\title{
Jamming Aspherical Cows
}

1. Universality of jamming of nonspherical particles.

Authors: C. Brito, H. Ikeda, P. Urbani, M. Wyart, F. Zamponi

Proceedings of the National Academy of Sciences of the U.S.A. 116, 11736-11741 (2018).

2. Translational and orientational glass transitions in the large-dimensional limit : a generalized replicated liquid theory and an application to patchy colloids

Authors: H. Yoshino

arXiv:1807.04095

\section{Recommended with a Commentary by Patrick Charbonneau, Duke University}

Spheres might essentialize many physical phenomena reasonably well, but not jamming. Donev and coworkers gave us a taste for the surpising impact of asphericity fifteen years ago by considering the packing properties of chocolate M\&Ms [2]. (See also [3].) Their initial finding that ellipsoids pack more densely than spheres was, however, less surprising than the hypostaticity of the associated contact networks [4]. In contrast to the perfect isostaticicy of amorphous packings of (frictionless) spheres [6], slightly aspherical objects violate Maxwell's criterion for mechanical stability. The higher-order mechanical constraints that make up the difference lead to exceptionally soft structural excitations [5].

While theoretical advances towards a first-principle description of jamming have since been sizable, they have exclusively concerned systems of spherical particles [1]. Yet if a comparable understanding of aspherical objects is still missing, it's not for lack of interest. Most realistic systems are far from spherically symmetric, (see Fig. 1), and even small deviations are strongly non-perturbative. Despite various rumors of a complete, first-principle derivation of the infinite-dimensional phase diagram of hard ellipsoids that could match in scope that of hard spheres, nothing concrete has emerged. Projects expected to produce results quickly were instead quietly dropped.

Without quite solving the problem of infinite-dimensional ellipsoids head on, Brito et al. provide different theoretical lenses through which to view the physics of jamming aspherical particles. The compressed presentation of the work (dictated by the publication format) might not please typical readers, but the minimal models and the stability arguments presented nonetheless deserve some attention. Their analysis reveals a marked difference between the jamming criticality of spherical and nonspherical particles. The isostaticity of the 

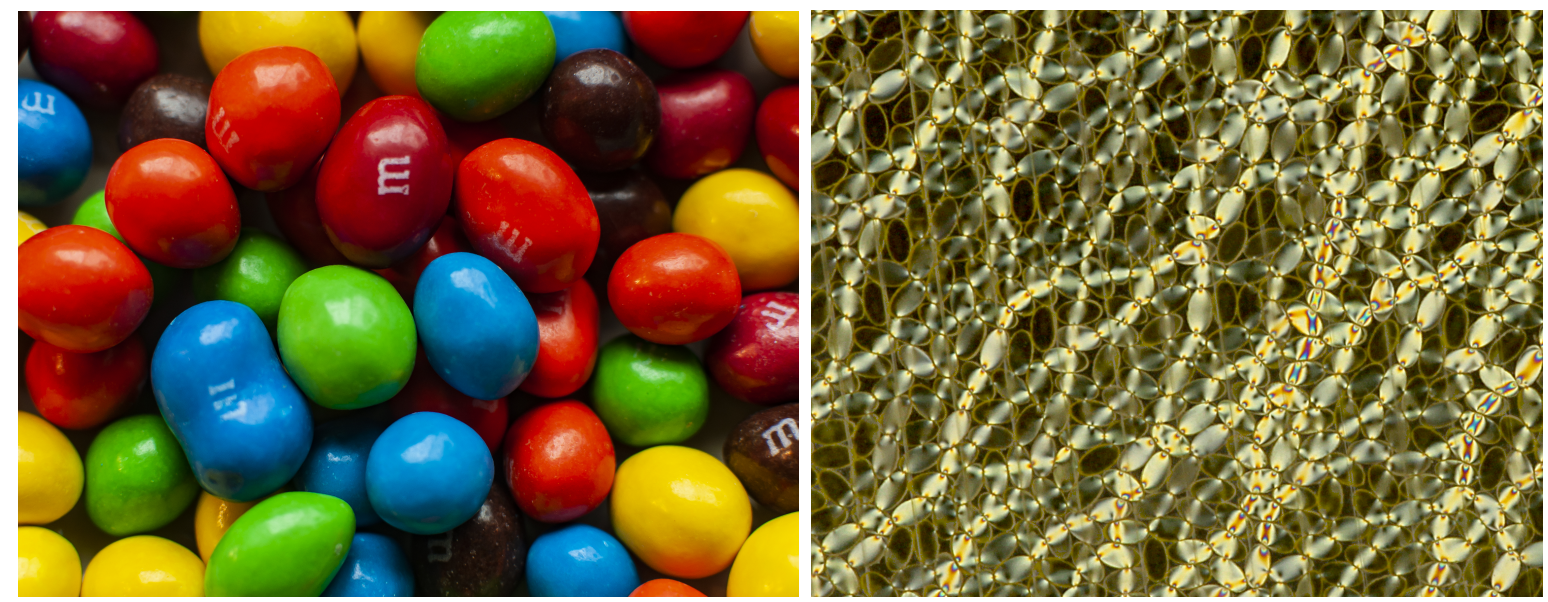

Figure 1: Jammed aspherical (frictional) particles: (left) popular candies; (right) photoelastic ellipses under stress seen through crossed polarizers. Photo credits: Irem Altan and LTB lab, respectively.

former leads to a power-law scaling of small gaps and weak forces, while such singularities are absent from the latter. The analysis further makes highly nontrivial predictions about the spectrum of vibrational excitations around jamming. These nuggets are fairly robust and should thus be testable in numerical simulations, and maybe even in experiments.

However significant this advance, it still falls short of providing a complete phase diagram of (even slightly) aspherical particles. Features, such as the rheological coupling of translation and rotation in the dilatency of amorphous solids and the non-monotonic scaling of the jamming threshold thus (mostly [7]) remain microscopically unexplained. A theoretical question of particular interest is whether some or any of these features emerge from lowdimensional geometry or generically appear in mean-field, infinite-dimensional descriptions (see [1] for similar considerations in hard spheres). This guidance would provide a strong basis for settling whether ordering of some type plays a role in the materials properties of amorphous solids. A more materials-centric concern is whether these couplings depend on the degree of thermalization. Such insights could have a genuine impact on the optimization and design of glasses obtained by vapor deposition, for instance.

The latest and most promising theoretical effort towards a complete description is that of Yoshino, who in his recent preprint develops a replicated liquid description of axially symmetric particles. This particular work constructs a first-principle theory of anisotropic attraction (i.e., attractive patchy spherical cows), but not of apshericity proper. A genuine breakthrough will hopefully soon follow. Some of us are eager for a first spherical harmonic deformation of the venerable sphere. Even if it's not quite like doing away with the frictionless approximation or introducing polymer chain connectivity from first principles, it's an important first symmetry to kick out.

This Commentary is dedicated to my late colleague Bob Behringer, who was deeply interested in asphericity these last few years (Fig. 1). This Commentary was supported by a grant from the Simons Foundation (\#454937) and by the National Science Foundation under Grant No. NSF PHY-1748958. 


\section{References}

[1] P. Charbonneau et al., Glass and Jamming Transitions: From Exact Results to FiniteDimensional Descriptions, Ann. Rev. Condens. Matter Phys. 8, 265-288 (2017).

[2] A. Donev et al., Improving the density of jammed disordered packings using ellipsoids, Science 303, 990-993 (2004).

[3] D. Frenkel, The tetrahedral dice are cast ... and pack densely, Physics 3, 37 (2010).

[4] A. Donev, R. Connelly, F. H. Stillinger, S. Torquato, Underconstrained jammed packings of nonspherical hard particles: Ellipses and ellipsoids. Physical Review E 75, 051304 (2007).

[5] M. Mailman, C. F. Schreck, C. S. O'Hern, B. Chakraborty, Jamming in Systems Composed of Frictionless Ellipse-Shaped Particles, Physical Review Letters 102, 255501 (2009).

[6] C. S. O'Hern, L. E. Silbert, A. J. Liu, S. R. Nagel, Jamming at zero temperature and zero applied stress: The epitome of disorder. Physical Review E 68, 011306 (2003).

[7] A. Baule, R. Mari, L. Bo, L. Portal, H. A. Makse, Mean-field theory of random close packings of axisymmetric particles. Nature Communication 4, (2013). 